\title{
Adenosquamous carcinoma of the uncinate process of the pancreas with synchronous gastrointestinal stromal tumor of the stomach: Case report and review of the literature
}

\author{
JIN-JIE HE ${ }^{1}$, KE-FENG DING ${ }^{1}$, LEI ZHENG $^{2}$, JING-HONG XU ${ }^{3}$, JUN LI $^{1}$, YU-LIAN WU ${ }^{4}$, \\ LI-FENG SUN ${ }^{1}$, DONG-ER ZHOU ${ }^{1}$ and SHU ZHENG ${ }^{1}$ \\ ${ }^{1}$ Department of Surgical Oncology, The Second Affiliated Hospital, Zhejiang University School of Medicine, Hangzhou, \\ Zhejiang 310009, P.R. China; ${ }^{2}$ Department of Oncology and Surgery, The Skip Viragh Center for Pancreatic Cancer, \\ The Sol Goldman Pancreatic Cancer Center, The Johns Hopkins University School of Medicine, Baltimore, \\ MD 21231, USA; Departments of ${ }^{3}$ Pathology and ${ }^{4}$ General Surgery, The Second Affiliated Hospital, \\ Zhejiang University School of Medicine, Hangzhou, Zhejiang 310009, P.R. China
}

Received June 11, 2012; Accepted August 29, 2012

DOI: $10.3892 / \mathrm{ol} .2012 .902$

\begin{abstract}
Recently, the coexistence of gastrointestinal stromal tumors (GISTs) with other neoplasms has been studied with increasing frequency. Coexistence of pancreatic cancer with GISTs remains a rarity; however, here, we report a very rare case of adenosquamous carcinoma (ASC) of the uncinate process of the pancreas with synchronous GISTs of the stomach in a 62-year-old female. The patient presented with epigastric discomfort and vomiting. Radiographic imaging revealed two masses; one located at the body of the stomach and the other located at the uncinate process of the pancreas. Intraoperatively, a fine needle aspiration biopsy was conducted in the uncinate process of the pancreas, which revealed the malignancy of the masses. A pancreaticoduodenectomy and partial gastrectomy were then conducted, and subsequent pathological examinations identified an ASC of the pancreas and a GIST of the stomach. In our case, contrary to the majority of previous cases of synchronous GISTs and other malignancies, GIST was not an incidental finding. The initial suspicion on the GIST as the underlying cause of clinical symptoms led to the discovery of the ASC of the uncinate process of the pancreas.
\end{abstract}

Correspondence to: Dr Li-feng Sun or Professor Ke-feng Ding, Department of Surgical Oncology, The Second Affiliated Hospital, Zhejiang University School of Medicine, 88 Jiefang Road, Hangzhou, Zheijang 310009, P.R. China

E-mail: sunlifengzju@gmail.com

E-mail: dingkefeng@126.com

Key words: adenosquamous carcinoma, gastrointestinal stromal tumor, synchronous cancer, uncinate process of the pancreas

\section{Introduction}

Recently, the synchronous occurrence of primary neoplasms has been studied with increasing frequency, which may be due to the improved preoperative imaging capabilities. The coexistence of gastrointestinal stromal tumors (GISTs) with other gastrointestinal or non-gastrointestinal malignancies has also been studied with increasing frequency, although the occurrence remains rare (1). It has been identified that the overall frequency of second tumors in different sites among patients with GISTs ranges between 4.5 and $18.6 \%$, with a mean of $13 \%$ (2).

GISTs are the most common type of mesenchymal tumor of the gastrointestinal tract, which most likely arise from precursor interstitial cells of Cajal. The majority of GISTs occur as a single lesion. They are common in the stomach (60-70\% of all cases) and small intestine (30\%), while they rarely occur in the rectum (5\%), esophagus, colon, pancreas, appendix, omentum, mesentery and retroperitoneum (3). The best survival predictors are the size, location and mitotic rate of the tumor.

Pancreatic cancer is a common gastrointestinal malignancy. Ductal adenocarcinoma accounts for $80-85 \%$ of pancreatic tumors, while adenosquamous carcinoma (ASC) of the pancreas is a rare, aggressive variant of pancreatic carcinoma with a worse prognosis. Its exact incidence is not known; however, autopsy and surgical specimen findings suggest that this lesion accounts for $1-4 \%$ of all exocrine malignancies of the pancreas (4).

The uncinate process is a hook-like extension of the lower part of the pancreatic head that projects medially and wraps around the superior mesenteric vessels. Carcinoma in the uncinate process of the pancreas (CUPP) appears to be less common than that in other parts of the pancreas. Previous studies identify an incidence rate of $2.5 \%$ (3/119) $(5), 7.7 \%$ (39/506) (6) and $10.7 \%$ (6/56) (7) of pancreatic malignancies. 
To the best of our knowledge, only one case in the literature demonstrated coexistence of ASC of the head of the pancreas with GIST (8), and the small GIST in this patient was only incidentally identified following surgery. With approved patient consent, we report a case of ASC of the uncinate process of the pancreas with the synchronous occurrence of large symptomatic GIST. 1. The study was approved by the Ethics Committee of The Second Affiliated Hospital, Zhejiang University School of Medicine, Hangzhou, China.

\section{Case report}

A 62-year-old female presented with a chief complaint of recurrent epigastric discomfort and progressively worsening vomiting for two weeks. The patient had experienced anorexia and weight loss for several months, but had no significant past medical history. The patient's family history was significant for pancreatic cancer from her mother, but no other malignancies were present in her other immediate family members. On admission, the patient's vital signs were stable and a mass of approximately $10 \mathrm{~cm}$ was palpated in the upper quadrant area of the abdomen. The remainder of the examination was unremarkable.

The complete blood count identified a white cell count of 6,900 cells $/ \mathrm{mm}^{3}$ (73.4\% neutrophils), hemoglobin count of $135 \mathrm{~g} / \mathrm{dl}$ and a platelet count of 181,000 platelets $/ \mathrm{mm}^{3}$. The blood chemistry examination revealed $23.0 \mu \mathrm{mol} / 1$ total bilirubin, $9.6 \mu \mathrm{mol} / 1$ direct bilirubin, $74 \mathrm{U} / 1$ aspartate transaminase (AST) and $102 \mathrm{U} / 1$ alanine aminotransferase (ALT). The carbohydrate antigen 19-9 (CA19-9) was slightly elevated to $78.4 \mathrm{U} / 1$ (normal range, 0-37 U/1), while carcinoembryonic antigen (CEA), $\alpha$-fetoprotein (AFP), carbohydrate antigen 24-2 (CA24-2), blood glucose, calcium and amylase levels were within normal ranges.

Computed tomography (CT) revealed a discrete mass measuring $8 \times 7 \mathrm{~cm}$ with notable enhancement in the body of stomach (Fig. 1). It also revealed a $4 \times 3-\mathrm{cm}$ lesion with an unclear boundary and mild enhancement in the uncinate process of pancreas and close to the horizontal part of the duodenum (Fig. 2). Ultrasonography of the liver, pancreas, spleen and gallbladder revealed no abnormality.

The initial impression from the CT scan was emphasized on the gastric mass as the lesion in the uncinate process of the pancreas was not well-defined. The gastric mass was suspected to be a GIST (Fig. 1), and the symptoms of bowel obstruction were suggested to be caused by the presumed GIST in the patient's stomach. However, an upper gastrointestinal series with meglumine diatrizoate was consistent with a partial bowel obstruction at the level of the third portion of duodenum. Therefore, the case was discussed at the multi-disciplinary team (MDT) conference, and it was recommended to intraoperatively explore the pancreas in addition to the resection of the gastric mass.

Intraoperatively, no liver metastasis or peritoneal dissemination was identified. A solid, partially capsulated mass, measuring approximately $8 \times 7 \times 7 \mathrm{~cm}$, was identified in the body of the stomach near the lesser curvature. Another solid mass was also revealed in the uncinate process of the pancreas, which had horizontally infiltrated part of duodenum and closely adhered to the inferior vena cava for $\sim 4 \mathrm{~cm}$ (Fig. 2).

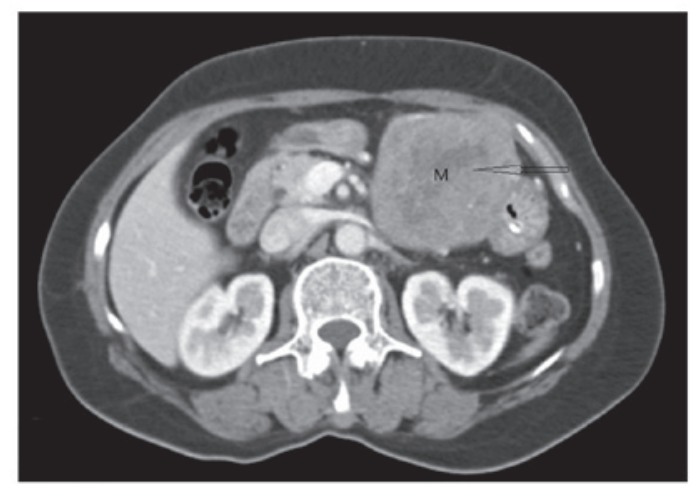

Figure 1. Abdominal CT scan revealing a tumor in the body of stomach (arrow). CT, computed tomography.

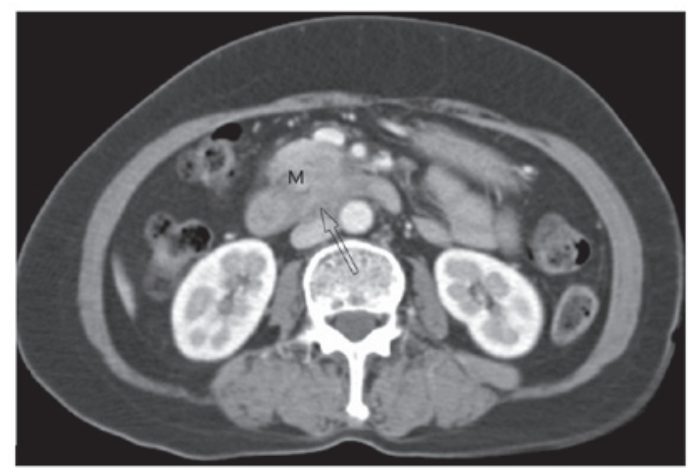

Figure 2. Abdominal CT scan revealing a tumor in the uncinate process of pancreas with an unclear boundary, which had horizontally infiltrated part of the duodenum and closely adhered to the inferior vena cava (arrow). CT, computed tomography.

A fine needle aspiration biopsy was conducted in the uncinate process of the pancreas and revealed the adenocarcinoma. Therefore, a pancreaticoduodenectomy (PD) was conducted, which included resection of the gastric mass and partial resection of the inferior vena cava.

The pathological specimen was composed of the head of the pancreas, distal portion of the stomach, common bile duct, duodenum and gallbladder. Microscopically, the gastric tumor tissue was comprised of spindle cells with coagulative necrosis and a mitotic rate of $\leq 5 / 50$ within a high power field (HPF; Fig. 3A). The tumor cells were immunoreactive for CD117 (Fig. 3B), supporting the diagnosis of GIST. The Union for International Cancer Control tumor-node-metastasis (UICC TNM) pathological staging of this GIST was pT3 (stage IB), and its National Institute of Health (NIH) risk stratification was intermediate. The pancreatic tumor was comprised of poorly differentiated cells with vascular and perineural invasion and infiltrating submucosa of duodenum (Fig. 4A). The tumor cells in the pancreas were immunoreactive for cytokeratin 5/6 (CK5/6), p63 and CAM5.2, supporting the diagnosis of ASC (Fig. 4B-D). None of the nine peripancreatic lymph nodes were involved. Ultimately, the pathological TNM staging was pT3NOMO.

The postoperative course was uneventful. The patient declined any further adjuvant therapy, and 6 months following 

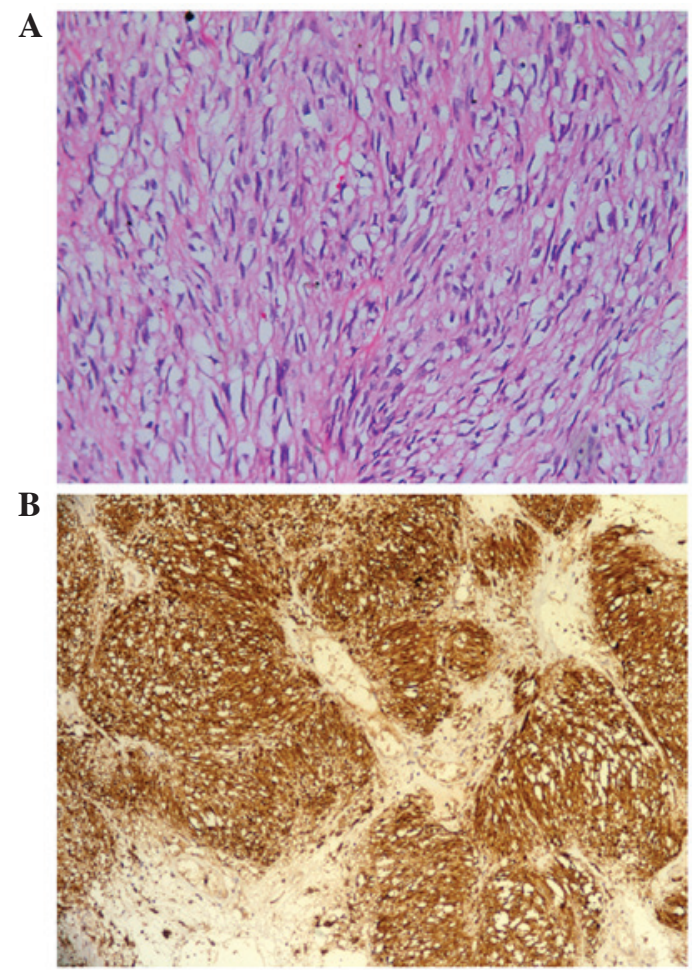

Figure 3. (A) Medium-power review of the gastric biopsy specimen identifiying a soft tissue mass consisting of a spindle cell neoplasm. (B) Positive immunohistochemistry staining for c-kit (CD117) was consistent with the diagnosis of GIST (magnification, x20). GIST, gastrointestinal stromal tumor.

therapy the patient remains healthy and has experienced no tumor recurrence.

\section{Discussion}

GISTs are the most common type of mesenchymal neoplasm of the gastrointestinal tract. Primary GISTs are usually solitary, but may occur in groups, e.g. Carney's triad or when associated with neurofibromatosis. Without the presence of Carney's triad or neurofibromatosis syndromes, synchronous occurrence of a GIST with a tumor of different histological type is rare and has been documented in the literature mainly in the form of case reports. Malignances of the gastrointestinal tract are the most commonly accompanied neoplasms; however, there are few previous studies concerning synchronous pancreatic cancer and GIST in the stomach $(8,9)$. Liu et al reported that the most common epithelial tumors associated with GIST in their series were esophageal squamous cell carcinomas (1.13\%), followed by gastric $(0.53 \%)$, pancreatic $(0.38 \%)$ and colorectal $(0.03 \%)$ adenocarcinomas (9).

Compared with a GIST alone, synchronous GISTs have various clinical manifestations. Agaimy and Wuensch analyzed a series of 97 cases of surgically resected GIST and revealed that the majority of GISTs in their series were benign or low-risk and appeared to be innocent bystanders (2). Liszka et al identified that these GISTs tended to be an incidental findings during surgery, and were most commonly localized in the small intestine (10). In our case, the GIST was associated with an intermediate risk and located in the stomach.
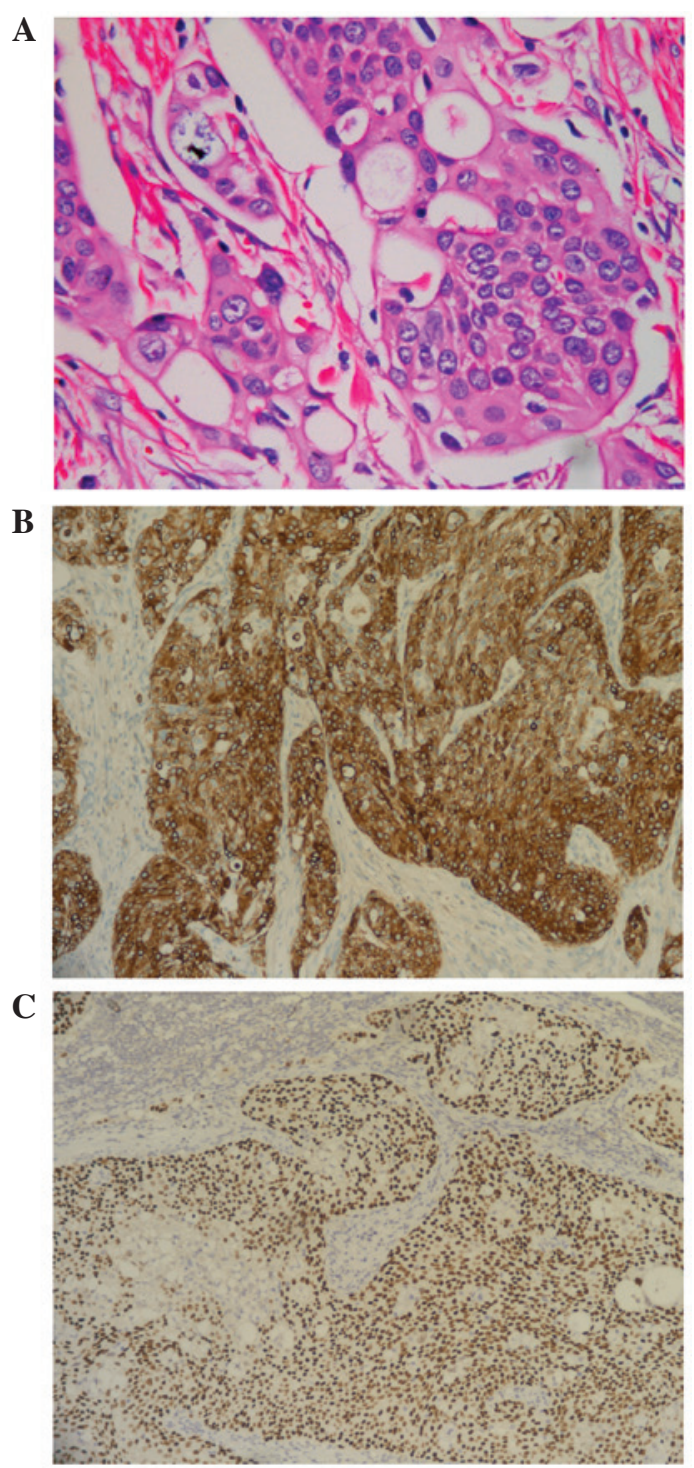

D

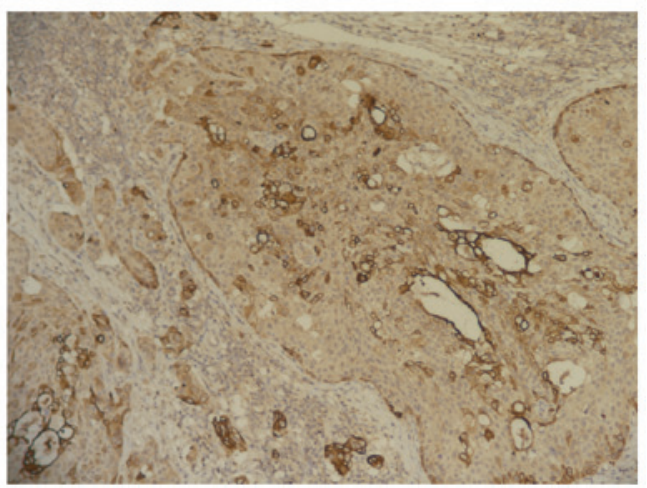

Figure 4. (A) Microphotograph of CUPP identifiying the intercellular bridge (the feature of squamous carcimoma) and mucus (magnification, x40). Subsequent images show positive immunohistochemistry staining for (B) CK5/6, (C) P63 and (D) CAM5.2, consistent with the diagnosis of adenosquamous carcimoma (magnification, $\mathrm{x} 20$ ). CUPP, carcinoma in the uncinate process of the pancreas.

ASC of the pancreas is also referred to as adenoacanthoma, mixed squamous adenocarcinoma and mucoepidermoid carcinoma. Radiographically, ASC cannot be distinguished from adenocarcinoma (11); thus, a pathological diagnosis may be made through biopsy or during surgery. Few patients undergo 
surgical resection as the majority of patients have stage IV disease at the time of presentation. When resected, ASC is frequently associated with positive lymph nodes, vascular and perineural invasion and poor tumor cell differentiation (12).

Patients with ASC have a worse survival rate compared with those with adenocarcinoma. Smit et al demonstrated that the average survival of 72 patients with ASC of the pancreas was 5.7 months, and only five patients survived for longer than one year (13). Kardon et al identified that the overall survival was 12.5 months in patients treated with curative resection and adjuvant chemotherapy, and was 3.0 months in patients who received no chemotherapy or received palliative chemotherapy (14). To date, surgical resection offers the only chance for a cure. Boyd et al recently revealed that the resectability is the strongest predictor of survival in ASC (15). Katz et al demonstrated an improvement in survival with the use of palliative radiation and chemotherapy; however, the benefit of adjuvant chemoradiation was not supported by this study (16). There is no current standard of chemotherapy regimens for patients with ASC, but it has been suggested that ASC of the pancreas is sensitive to platinum-containing chemotherapy regimens $(17,18)$.

The preoperative pathological diagnosis of ASC is often difficult. The carcinoma arising from the uncinate process has unique clinical manifestations due to its anatomical location, and the clinical presentation of CUPP is often late as a result of the lack of obstructive jaundice as a presenting feature. More common symptoms including abdominal pain and weight loss occur in up to $70 \%$ of all cases. Due to its tendency to cause duodenal obstruction, vomiting, as observed in our patient, is also a common clinical presentation. Due to the anatomical position of the uncinate process, ultrasound imaging of this area is likely to be obscured by the overlying bowel; therefore, CT is the main diagnostic method (6). Additionally, CUPP commonly involves superior mesentery vessels, making it unresectable or leading to margin-positive resection.

The overall survival in CUPP is less than that of the adenocarcinoma in the head of the pancreas. Ye et al identified a one-year survival rate of $37.7 \%$ and a 5-year survival rate of $5.6 \%$ for all stages of CUPP (19). Li et al revealed that resected CUPPs had a median survival of 17 months. Those patients who did not have venous resection had a median survival of 19 months, while those with venous resection had a median survival of 13 months (20). Thus, a delay in clinical presentation and the anatomical location of CUPP in relation to the retroperitoneum and mesenteric vessels appears to account for lower resection rates and reduced overall survival.

Contrary to the majority of previous cases of synchronous GISTs and other malignancies $(9,10)$, and the other case report of synchronous GIST and pancreatic adenocarcinoma (8), in our case, GIST was not an incidental finding. Our initial impression was a symptomatic GIST; however, following reevaluation via a $\mathrm{CT}$ scan with a multidisciplinary approach, a pancreatic adenocarcinoma was suspected. Thus, the intraoperative exploration of the pancreas and the pancreatoduodectomy was preoperatively planned.

\section{References}

1. Efstathios P, Athanasios P, Papaconstantinou I, et al: Coexistence of gastrointestinal stromal tumor (GIST) and colorectal adenocarcinoma: a case report. World J Surg Oncol 5: 96, 2007.

2. Agaimy A and Wuensch PH: Gastrointestinal stromal tumours in patients with other-type cancer: a mere coincidence or an etiological association? A study of 97 GIST cases. Z Gastroenterol 43: 1025-1030, 2005

3. Miettinen M and Lasota J: Gastrointestinal stromal tumors definition, clinical, histological, immunohistochemical, and molecular genetic features and differential diagnosis. Virchows Arch 438: 1-12, 2001.

4. Madura JA, Jarman BT, Doherty MG, Yum MN and Howard TJ: Adenosquamous carcinoma of the pancreas. Arch Surg 134: 599-603, 1999.

5. Yamaguchi K: Carcinoma of the uncinate process of the pancreas with a peculiar clinical manifestation. Am J Gastroenterol 87: 1046-1050, 1992.

6. Birk D, Schoenberg MH, Gansauge F, Formentini A, Fortnagel G and Beger HG: Carcinoma of the head of the pancreas arising from the uncinate process. Br J Surg 85: 498-501, 1998.

7. Suzuki T, Kuratsuka H, Uchida K, Matsumoto Y and Honjo I: Carcinoma of the pancreas arising in the region of the uncinate process. Cancer 30: 796-800, 1972.

8. Dasanu CA, Mesologites T and Trikudanathan G: Synchronous tumors: adenosquamous carcinoma of pancreas and GIST of stomach. J Gastrointest Cancer 42: 186-189, 2011.

9. Liu YJ, Yang Z, Hao LS, Xia L, Jia QB and Wu XT: Synchronous incidental gastrointestinal stromal and epithelial malignant tumors. World J Gastroenterol 15: 2027-2031, 2009.

10. Liszka L, Zielinska-Pajak E, Pajak J, Golka D and Huszno J: Coexistence of gastrointestinal stromal tumors with other neoplasms. J Gastroenterol 42: 641-649, 2007.

11. Okabayashi T and Hanazaki K: Surgical outcome of adenosquamous carcinoma of the pancreas. World J Gastroenterol 14: 6765-6770, 2008.

12. Voong KR, Davison J, Pawlik TM, et al: Resected pancreatic adenosquamous carcinoma: clinicopathologic review and evaluation of adjuvant chemotherapy and radiation in 38 patients. Hum Pathol 41: 113-122, 2010.

13. Smit W, Mathy JP and Donaldson E: Pancreatic cytology and adenosquamous carcinoma of the pancreas. Pathology 25: 420-422, 1993

14. Kardon DE, Thompson LD, Przygodzki RM and Heffess CS: Adenosquamous carcinoma of the pancreas: a clinicopathologic series of 25 cases. Mod Pathol 14: 443-451, 2001.

15. Boyd CA, Benarroch-Gampel J, Sheffield KM, Cooksley CD and Riall TS: 415 patients with adenosquamous carcinoma of the pancreas: a population-based analysis of prognosis and survival. J Surg Res 174: 12-19, 2011.

16. Katz MH, Taylor TH, Al-Refaie WB, et al: Adenosquamous versus adenocarcinoma of the pancreas: a population-based outcomes analysis. J Gastrointest Surg 15: 165-174, 2011.

17. Aurilio G, Macarulla T, Ramos JF, Fazio N, Nolè F and Iglesias C: Successful treatment with GEMOX in patient with metastatic pancreatic adenosquamous carcinoma. Tumori 97: 239-242, 2011.

18. Wilkowski R, Boeck S, Ostermaier S, et al: Chemoradiotherapy with concurrent gemcitabine and cisplatin with or without sequential chemotherapy with gemcitabine/cisplatin vs chemoradiotherapy with concurrent 5-fluorouracil in patients with locally advanced pancreatic cancer - a multi-centre randomised phase II study. Br J Cancer 101: 1853-1859, 2009.

19. Ye C, Xi PC and Hu XG: Clinical analysis of uncinate process carcinoma of the pancreas. Hepatobiliary Pancreat Dis Int 2: 605-608, 2003.

20. Li S, Pei YQ, Du FT, et al: Surgical treatment for uncinate process carcinoma of the pancreas. Hepatobiliary Pancreat Dis Int 1: 592-594, 2002. 